\title{
Integrated bioinformatics analysis revealing independent prognostic long non-coding RNAs DNAH17-AS1 and RP11-400N13.2 and their potential oncogenic roles in colorectal cancer
}

\author{
WEN ZHOU ${ }^{1 *}$, BOYU PAN ${ }^{2,3 *}$ and LIREN LIU ${ }^{2,3}$ \\ ${ }^{1}$ Clinical Laboratory, Tianjin Hospital, Tianjin 300211; ${ }^{2}$ Department of Gastrointestinal Cancer Biology, \\ ${ }^{3}$ National Clinical Research Center for Cancer, Tianjin Medical University Cancer Institute and Hospital,
} Key Laboratory of Cancer Prevention and Therapy, Tianjin Clinical Research Center for Cancer, Tianjin 300060, P.R. China

Received February 5, 2019; Accepted June 24, 2019

DOI: $10.3892 / \mathrm{ol} .2019 .10730$

\begin{abstract}
The aberrant expression of long non-coding RNAs (lncRNAs) has been associated with a variety of malignancies, including colorectal cancer (CRC); however, the key lncRNAs associated with patient prognosis and their biological roles in CRC are yet to be determined. The aim of the present study was to determine the key lncRNAs associated with patient prognosis as well as their biological roles in CRC. Therefore, a dataset from The Cancer Genome Atlas containing the IncRNA expression data of $521 \mathrm{CRC}$ and normal colorectal mucosal tissues, as well as the corresponding clinical data, were screened. A total of 1,180 significantly differentially expressed lncRNAs were associated with CRC as determined by t-tests in edgeR. Kaplan-Meier analysis revealed that 56 of the 1,180 lncRNAs were associated with overall survival (OS); 7 of the 56 lncRNAs were identified as key lncRNAs associated with the Tumor-Node-Metastasis stage of CRC by Kruskal-Wallis test. Subsequent univariate and multivariate Cox regression analyses of the 7 lncRNAs revealed 2 lncRNAs, DNAH17-AS1 and RP11-400N13.2, as potential independent prognostic factors for the OS of patients with CRC. Furthermore, the expression
\end{abstract}

Correspondence to: Professor Liren Liu, Department of Gastrointestinal Cancer Biology, Tianjin Medical University Cancer Institute and Hospital, Huanhuxi Road, Hexi, Tianjin 300060, P.R. China

E-mail: liuliren@tmu.edu.cn

*Contributed equally

Abbreviations: lncRNAs, long non-coding RNAs; CRC, colorectal cancer; OS, overall survival; PCGs, protein-coding genes; GO, Gene Ontology; KEGG, Kyoto Encyclopedia of Genes and Genomes

Keywords: DNAH17-AS1, RP11-400N13.2, colorectal cancer, long non-coding RNA, bioinformatics analysis, independent prognostic biomarker, oncogenic levelsof these 2 lncRNAs were significantly upregulated in CRC compared with those in normal tissues, which suggested that they may serve an oncogenic role in CRC. In addition, networks comprising the 2 lncRNAs and their respective co-expressed protein-coding genes (PCGs) were constructed using cor.test in R. Gene Ontology and Kyoto Encyclopedia of Genes and Genomes enrichment analyses of these PCGs were conducted; DNAH17-AS1- and RP11-400N13.2-associated PCGs were reported to be involved in G-protein coupling-related functions. Thus, these independent prognostic lncRNAs and their associated functions identified in the present study may provide novel insight into potential prognostic biomarkers and therapeutic targets for the treatment of CRC.

\section{Introduction}

Colon cancer is the most common type of tumor of the gastrointestinal tract, and ranks as the third highest cause of cancer-associated mortality worldwide (1). The etiology and pathogenesis of colon cancer are complex and are associated with various factors, such as diet- and lifestyle-associated genetic and epigenetic changes (2). Recent advances in the treatment of colon cancer have been reported, including surgery combined with chemotherapy, radiofrequency ablation or targeted therapy; however, the rate of postoperative recurrence remain at $\sim 50 \%$, leading to a poor overall survival (OS) for the patients with colon cancer (3). Therefore, there is an urgent need to identify novel biomarkers and potential therapeutic targets for this deadly disease (4).

Long non-coding RNAs (IncRNAs), which are $>200$ nucleotides in length, have been reported to act as key regulators of various biological processes; the aberrant expression of lncRNAs are associated with several diseases, including cancer (5-9). Accumulating evidence has suggested that lncRNAs could serve as potential biomarkers for the early diagnosis, prognosis and prediction of metastasis for various types of malignancy (10-15).

In recent years, with advances in bioinformatics and interdisciplinary studies involving the development of a series 
of computational methods and software tools for the analysis of extensive biological data, numerous lncRNAs have been identified to be dysregulated in colon cancer. For instance, by a bioinformatic approach, a recent study classified Linc00659 as a novel oncogenic lncRNA involved in the tumorigenesis of colon cancer by modulating the progression of the cell cycle; downregulation of Linc00659 expression resulted in severe cell cycle arrest and enhanced the apoptosis of colon cancer cells (16). Similarly, based on bioinformatics analysis of The Cancer Genome Atlas (TCGA) and/or the Gene Expression Omnibus datasets, as well as subsequent experimental validation, metastasis-associated lung adenocarcinoma transcript 1 and small nuclear host gene 1 have been recently identified to be oncogenic lncRNAs, which may serve as potential diagnostic and therapeutic targets in colorectal cancer (CRC) (17-20). These results suggest the potential clinical value of lncRNAs in CRC; however, the lncRNAs associated with the prognosis and survival of patients, as well as their biological roles, require further investigation.

Therefore, the present study aimed to identify the key lncRNAs associated with their prognostic and biological roles using a comprehensive bioinformatics process. The gene expression datasets downloaded from The Cancer Genome Atlas (TCGA) database, which includes the corresponding survival and Tumor-Node-Metastasis (TNM) stage (21) status of patients with CRC, were utilized to construct a prognostic prediction system.

\section{Materials and methods}

TCGA CRC data mining and screening. The level 3 normalized lncRNA expression data of CRC, CRC gene expression data and corresponding clinical data were obtained from the TCGA database (https://cancergenome. nih.gov). The expression profiling platform RNA-seqv2 was used. No further normalizations were applied to the level 3 lncRNA expression profile data. A total of 521 samples were obtained, of which 480 were CRC tissues and 41 were adjacent normal tissues. The lncRNA expression profile of tumor and normal tissues was determined to screen for differentially expressed lncRNAs using edgeR (http://www. bioconductor.org/packages/release/bioc/html/edgeR.html; R software; version 3.4.2; Bell Laboratories) with thresholds of $\mid \log 2$ [fold-change (FC)] $>2.0$ and adjusted P-value [false discovery rate $(\mathrm{FDR})]<0.05$. A volcano plot was generated using theplot function in R (https://www.rdocumentation. org/packages/graphics/versions/3.6.0/topics/pl; R software; version 3.4.2; Bell Laboratories).

Survival analysis. Kaplan-Meier analysis followed by a log-rank test was performed to assess the OS between low- and high-lncRNA expression groups using the R package 'survival' (https://cran.r-project.org/web/packages/survival/index.html; $\mathrm{R}$ software; version 3.4.2; Bell Laboratories). The KruskalWallis test was used to evaluate the association between tumor stage. The staging system of colon cancer using UICC/AJCC (7th edition) (21), and the IncRNAs that were significantly associated with OS. Additionally, univariate and multivariate Cox regression analyses were used to evaluate the association between the expression levels of IncRNAs and the OS of patients with CRC, and to identify independent prognostic values of lncRNAs. $\mathrm{P}<0.05$ was considered to indicate a statistically significant difference.

Analysis of co-expressed protein-coding genes (PCGs). To determine the association between IncRNAs and co-expressed PCGs, the Pearson correlation coefficients (r) of the lncRNAs and PCGs were calculated using the cor.test function in R. The PCGs with $|r|>0.4$ and $\mathrm{P}<0.001$ were considered as lncRNA-associated PCGs.

Functional and pathway enrichment analyses. The identified co-expressed PCGs were further investigated using clusterProfiler R package (http://www.bioconductor. org/packages/release/bioc/html/clusterProfiler.html; R software; version 3.4.2; Bell Laboratories), including functional Gene Ontology (GO) (22) and Kyoto Encyclopedia of Genes and Genomes (KEGG) (23) pathway enrichment analyses. $\mathrm{P}<0.05$ was considered to indicate a statistically significant difference.

\section{Results}

Identification of significantly differentially expressed lncRNAs in CRC. In a preliminary screening, the data of $480 \mathrm{CRC}$ and 41 adjacent normal colorectal mucosal tissues were extracted from the symbol matrix. A total of 1,180 significant differentially expressed lncRNAs were identified with $\mid \operatorname{logFCl}>2$ and FDR $<0.05$, of which 916 were upregulated and 264 were downregulated. A volcano plot of the identified lncRNAs was constructed (Fig. 1). The top 10 upregulated and downregulated lncRNAs are presented in Tables I and II.

Analysis of significant differentially expressed lncRNAs in CRC samples associated with OS and pathological stages. To investigate the association between lncRNA expression and OS, the expression profile of the 1,180 lncRNAs in tumor samples were determined, of which 56 lncRNAs were associated with OS, as determined by Kaplan-Meier analysis (Table III). The top 10 lncRNAs significantly associated with OS (P<0.05) were RP11-108K3.2, RP11-815M8.1, LINC01836, AC079612.1, LINC01354, RBAKDN, RP11-400N13.2, RP1-142L7.9, AFAP1-AS1 and LINC01655 (Fig. 2).

The association between clinical stages (UICC/AJCC 7th Edition) (21) and the 56 lncRNAs associated with OS was determined via a Kruskal-Wallis test. The results demonstrated that 7 lncRNAs were identified as key lncRNAs associated with the Tumor-Node-Metastasis (TNM) stages of colon cancer, including DNAH17-AS1, RP11-429J17.5, RP11-742B18.1, RP11-400N13.2, LL22NC03-N14H11.1, LINC01836 and HOTAIR (Table IV; Fig. 3). The detailed information of the patients at each TNM stage is presented in Table SI. Notably, these 7 lncRNAs were upregulated in colon cancer tissues compared with adjacent normal tissues, suggesting that they may serve a tumorigenic role in the initiation and progression of CRC.

Identification of independent prognostic lncRNAs in CRC. In order to detect potential independent prognostic IncRNAs in patients with $\mathrm{CRC}$, univariate and multivariate Cox regression 
Table I. Top 10 upregulated lncRNAs with significantly different expression between tumor and normal tissues in The Cancer Genome Atlas colon cancer data.

\begin{tabular}{llccc}
\hline lncRNA & Ensembl_Gene_ID & logFC & P-value \\
\hline PVT1 & ENSG00000249859 & 2.58 & $1.70 \times 10^{-67}$ & $4.23 \times 10^{-65}$ \\
CCAT1 & ENSG00000247844 & 4.47 & $1.68 \times 10^{-59}$ & $2.51 \times 10^{-57}$ \\
BLACAT1 & ENSG00000281406 & 5.12 & $3.88 \times 10^{-59}$ & $5.59 \times 10^{-57}$ \\
LINC02163 & ENSG00000251026 & 7.08 & $1.19 \times 10^{-54}$ & $1.37 \times 10^{-52}$ \\
CRNDE & ENSG00000245694 & 4.58 & $1.30 \times 10^{-52}$ & $1.31 \times 10^{-50}$ \\
MAFG-AS1 & ENSG00000265688 & 2.88 & $7.82 \times 10^{-51}$ & $7.51 \times 10^{-49}$ \\
RP5-884M6.1 & ENSG00000228742 & 6.31 & $3.30 \times 10^{-50}$ & $3.69 \times 10^{-49}$ \\
CASC19 & ENSG00000254166 & 4.51 & $7.64 \times 10^{-50}$ & $6.65 \times 10^{-48}$ \\
RP5-1120P11.1 & ENSG00000237686 & 4.41 & $2.64 \times 10^{-48}$ & $2.17 \times 10^{-46}$ \\
AC007128.1 & ENSG00000229970 & 4.48 & &
\end{tabular}

lncRNA, long non-coding RNA; FC, fold-change; FDR, false discovery rate.

Table II. Top 10 downregulated lncRNAs with significantly different expression between tumor and normal tissues in The Cancer Genome Atlas colon cancer data.

\begin{tabular}{lllll}
\hline lncRNA & Ensembl_Gene_ID & logFC & P-value \\
\hline XXbac-B476C20.9 & ENSG00000225335 & -2.72 & $2.14 \times 10^{-173}$ & $1.60 \times 10^{-169}$ \\
CDKN2B-AS1 & ENSG00000240498 & -5.21 & $9.05 \times 10^{-140}$ & $3.39 \times 10^{-136}$ \\
LINC01645 & ENSG00000224968 & -4.60 & $6.90 \times 10^{-127}$ & $1.72 \times 10^{-123}$ \\
PP7080 & ENSG00000188242 & -3.48 & $3.24 \times 10^{-124}$ & $6.07 \times 10^{-121}$ \\
XXyac-YM21GA2.7 & ENSG00000214888 & -5.69 & $7.06 \times 10^{-120}$ & $1.06 \times 10^{-116}$ \\
RP11-1090M7.1 & ENSG00000265489 & -4.48 & $1.49 \times 10^{-116}$ & $1.85 \times 10^{-113}$ \\
RP11-396O20.2 & ENSG00000254645 & -5.01 & $4.65 \times 10^{-114}$ & $1.83 \times 10^{-111}$ \\
AC007182.6 & ENSG00000224721 & -4.67 & $2.71 \times 10^{-105}$ & $4.35 \times 10^{-105}$ \\
AC106869.2 & ENSG00000226087 & -4.18 & $1.18 \times 10^{-96}$ & $2.25 \times 10^{-102}$ \\
LINC00682 & ENSG00000245870 & -4.55 & $8.82 \times 10^{-94}$ \\
\hline
\end{tabular}

lncRNA, long non-coding RNA; FC, fold-change; FDR, false discovery rate.

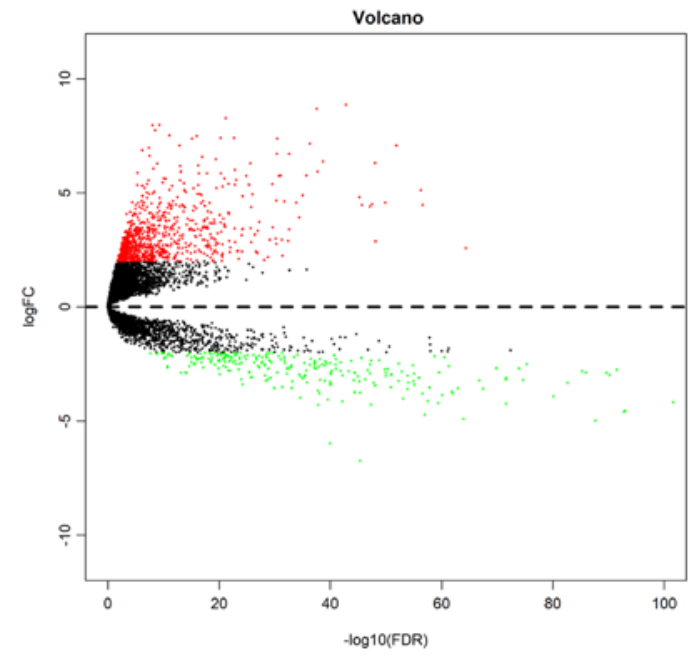

Figure 1. Volcano plot of significantly differentially expressed lncRNAs associated with colorectal cancer. Red, green and black dots represent upregulated, downregulated and non-differentially expressed lncRNAs, respectively. lncRNA, long non-coding RNA; FC, fold-change; FDR, false discovery rate. analyses of these 7 lncRNAs associated with TNM stage were performed. The association between the expression levels of lncRNAs and the OS of patients with colon cancer was explored using the R package 'survival'; 2 lncRNAs, DNAH17-AS1 and RP11-400N13.2, were identified to be independent prognostic factors for OS in patients with CRC $(\mathrm{P}<0.05$; Tables V and VI).

Analyses of PCGs co-expressed with lncRNAs DNAH17-AS1 and RP11-400N13.2. Analysis of the PCGs co-expressed with DNAH17-AS1 and RP11-400N13.2 was conducted using the cor.test function with thresholds of $|r|>0.4$ and $\mathrm{P}<0.001$. The results revealed that 1,048 PCGs were co-expressed with DNAH17-AS1 (Fig. 4A). Due to a large number of PCGs in DNAH17-AS1, only the top 100 genes with a lower P-value were presented in Fig. 4A. A total of 126 PCGs co-expressed with RP11-400N13.2 (Fig. 4B). The top 10 significant PCGs that were identified to be co-expressed with the 2 lncRNAs are listed in Table VII. 
Table III. IncRNAs significantly associated with OS.

\begin{tabular}{|c|c|c|c|c|c|}
\hline lncRNA & Ensembl_Gene_ID & $\mathrm{P}$-value & lncRNA & Ensembl_Gene_ID & P-value \\
\hline RP11-108K3.2 & ENSG00000259306 & $1.98646 \times 10^{-5}$ & LINC00461 & ENSG00000245526 & 0.04796558 \\
\hline RP11-815M8.1 & ENSG00000238042 & $2.78454 \times 10^{-5}$ & CTD-2600O9.1 & ENSG00000187185 & 0.048650792 \\
\hline LINC01836 & ENSG00000267530 & 0.000256940 & LEF1-AS1 & ENSG00000232021 & 0.049375626 \\
\hline AC079612.1 & ENSG00000196758 & 0.000535749 & FLJ16779 & ENSG00000275620 & 0.049920309 \\
\hline LINC01354 & ENSG00000231768 & 0.000985613 & & & \\
\hline RBAKDN & ENSG00000273313 & 0.001143877 & icRNA, long non-codin & RNA. & \\
\hline RP11-400N13.2 & ENSG00000228437 & 0.002268513 & & & \\
\hline RP1-142L7.9 & ENSG00000270661 & 0.003700521 & & & \\
\hline AFAP1-AS1 & ENSG00000272620 & 0.004000305 & Table IV. IncRNAs si & cantly associated $\mathrm{x}$ & umor clinical \\
\hline LINC01655 & ENSG00000227925 & 0.004380952 & & & \\
\hline RP11-10A14.5 & ENSG00000248538 & 0.004693149 & & & \\
\hline RP11-384P7.7 & ENSG00000260947 & 0.004845978 & cRNA & Ensembl_Gene_ID & P-value \\
\hline RP11-434D9.2 & ENSG00000249894 & 0.005561553 & & & \\
\hline RP11-742B18.1 & ENSG00000249001 & 0.007363036 & FLJ16779 & ENSG00000275620 & 0.049920309 \\
\hline CTC-327F10.4 & ENSG00000251320 & 0.008786437 & -AS1 & SG00000267432 & $0^{-5}$ \\
\hline AC064834.1 & ENSG00000224099 & 0.00890189 & 7.5 & 0000 & 0.0 \\
\hline ARHGEF26-AS1 & ENSG00000243069 & 0.008966797 & & El & \\
\hline RP3-380B8.4 & ENSG00000233064 & 0.009485845 & & $\mathrm{EN}$ & \\
\hline LINC01829 & ENSG00000236780 & 0.009974597 & 0 & $\mathrm{E}$ & \\
\hline GAS1RR & ENSG00000226237 & 0.013908392 & 6 & & \\
\hline RP11-84A19.4 & ENSG00000269967 & 0.014180465 & & $\mathrm{E}$ & 00989 \\
\hline LINC02043 & ENSG00000232233 & 0.01633 & \multirow{2}{*}{\multicolumn{3}{|c|}{ lncRNA, long non-coding RNA. }} \\
\hline LINC00922 & ENSG00000261742 & 0.016854765 & & & \\
\hline RP11-278L15.2 & ENSG00000243885 & 0.017325027 & \multirow{2}{*}{\multicolumn{3}{|c|}{$\begin{array}{l}\text { GO and KEGG enrichmentanalyses of the PCGs co-expressed } \\
\text { with DNAH17-ASI and RP11-400N13.2. To further inves- } \\
\text { tigate the potential roles of the two independent prognostic } \\
\text { lncRNAs identified in the present study, functional enrich- } \\
\text { ment analyses for their co-expressed PCGs were performed } \\
\text { using the clusterProfiler R package. The results indicated } \\
\text { that the PCGs co-expressed with DNAH17-AS1 were mainly } \\
\text { enriched in 'G-protein coupled receptor signaling pathway', } \\
\text { 'detection of chemical stimulus involved in sensory percep- } \\
\text { tion of smell', 'integral component of membrane', 'integral } \\
\text { component of plasma membrane', 'G-protein coupled receptor } \\
\text { activity' and 'olfactory receptor activity'. Collectively, } \\
\text { these PCGs were associated with G-protein coupling and } \\
\text { cell membrane function (Fig. 5A). Additionally, the PCGs } \\
\text { co-expressed with RP11-400N13.2 were mainly enriched in } \\
\text { 'G-protein coupled receptor signaling pathway', 'G-protein } \\
\text { coupled receptor activity', 'spermatogenesis', 'negative regula- } \\
\text { tion of endopeptidase activity' and 'endopeptidase inhibitor } \\
\text { activity', which are involved in G-protein coupling and endo- } \\
\text { peptidase function (Fig. 5B). Functional enrichment analysis } \\
\text { revealed a high level of involvement of the associated PCGs } \\
\text { inG-protein coupling, which suggested a crucial biological } \\
\text { function of DNAH17-AS1 and RP11-400N13.2. The detailed } \\
\text { information of the genes with G-protein-associated func- } \\
\text { tions co-expressed with DNAH17-AS1 and RP11-400N13.2 } \\
\text { is presented in Table SII. A total of } 3 \text { of these genes, } \\
5 \text { '-hydroxytryptamine receptor } 6 \text {, melanocortin } 5 \text { receptor and } \\
\text { prokineticin receptor } 2 \text {, were significantly associated with OS } \\
\text { (P<0.05; Fig. S1). }\end{array}$}} \\
\hline RP1-122P22.4 & ENSG00000268628 & 0.018 & & & \\
\hline RP11-366L20.2 & ENSG00000197301 & 0.019632443 & \multirow{27}{*}{\multicolumn{3}{|c|}{$\begin{array}{l}\text { GO and KEGG enrichmentanalyses of the PCGs co-expressed } \\
\text { with DNAH17-AS1 and RP11-400N13.2. To further inves- } \\
\text { tigate the potential roles of the two independent prognostic } \\
\text { lncRNAs identified in the present study, functional enrich- } \\
\text { ment analyses for their co-expressed PCGs were performed } \\
\text { using the clusterProfiler R package. The results indicated } \\
\text { that the PCGs co-expressed with DNAH17-AS1 were mainly } \\
\text { enriched in 'G-protein coupled receptor signaling pathway', } \\
\text { 'detection of chemical stimulus involved in sensory percep- } \\
\text { tion of smell', 'integral component of membrane', 'integral } \\
\text { component of plasma membrane', 'G-protein coupled receptor } \\
\text { activity' and 'olfactory receptor activity'. Collectively, } \\
\text { these PCGs were associated with G-protein coupling and } \\
\text { cell membrane function (Fig. 5A). Additionally, the PCGs } \\
\text { co-expressed with RP11-400N13.2 were mainly enriched in } \\
\text { 'G-protein coupled receptor signaling pathway', 'G-protein } \\
\text { coupled receptor activity', 'spermatogenesis', 'negative regula- } \\
\text { tion of endopeptidase activity' and 'endopeptidase inhibitor } \\
\text { activity', which are involved in G-protein coupling and endo- } \\
\text { peptidase function (Fig. 5B). Functional enrichment analysis } \\
\text { revealed a high level of involvement of the associated PCGs } \\
\text { inG-protein coupling, which suggested a crucial biological } \\
\text { function of DNAH17-AS1 and RP11-400N13.2. The detailed } \\
\text { information of the genes with G-protein-associated func- } \\
\text { tions co-expressed with DNAH17-AS1 and RP11-400N13.2 } \\
\text { is presented in Table SII. A total of } 3 \text { of these genes, } \\
5 \text { '-hydroxytryptamine receptor 6, melanocortin } 5 \text { receptor and } \\
\text { prokineticin receptor } 2 \text {, were significantly associated with OS } \\
\text { (P<0.05; Fig. S1). }\end{array}$}} \\
\hline DUXAP8 & ENSG00000206195 & 0.020361403 & & & \\
\hline СТB-181H17.1 & ENSG00000272219 & 0.020685373 & & & \\
\hline MIR31HG & ENSG00000171889 & 0.021365649 & & & \\
\hline $\mathrm{AC} 012531.25$ & ENSG00000260597 & 0.023942649 & & & \\
\hline FOXD3-AS1 & ENSG00000230798 & 0.024144909 & & & \\
\hline AC007128.1 & ENSG00000229970 & 0.027145232 & & & \\
\hline DNAH17-AS1 & ENSG00000267432 & 0.02735923 & & & \\
\hline LINC01833 & ENSG00000259439 & 0.027941465 & & & \\
\hline RP11-429J17.5 & ENSG00000254548 & 0.028089082 & & & \\
\hline LL22NC03-N14H11.1 & ENSG00000272872 & 0.031532618 & & & \\
\hline RP1-79C4.4 & ENSG00000271811 & 0.031571197 & & & \\
\hline CTB-186G2.4 & ENSG00000267375 & 0.031679272 & & & \\
\hline RP11-114H23.2 & ENSG00000258088 & 0.033221364 & & & \\
\hline LINC00484 & ENSG00000229694 & 0.035515205 & & & \\
\hline RP1-29C18.10 & ENSG00000212939 & 0.035961207 & & & \\
\hline AC073326.3 & ENSG00000228540 & 0.036298277 & & & \\
\hline RP11-126H7.4 & ENSG00000204049 & 0.036449014 & & & \\
\hline CTD-2619J13.13 & ENSG00000268307 & 0.037145687 & & & \\
\hline LINC01748 & ENSG00000226476 & 0.038383393 & & & \\
\hline RP11-532F6.3 & ENSG00000272463 & 0.042708274 & & & \\
\hline RP11-728G15.1 & ENSG00000256008 & 0.043149989 & & & \\
\hline LINC01060 & ENSG00000249378 & 0.045508319 & & & \\
\hline KCNQ1OT1 & ENSG00000269821 & 0.045529181 & & & \\
\hline LINC01996 & ENSG00000261863 & 0.046591159 & & & \\
\hline ELFN1-AS1 & ENSG00000236081 & 0.046625182 & & & \\
\hline HOTAIR & ENSG00000228630 & 0.047908786 & & & \\
\hline
\end{tabular}

Table III. Continued.

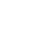

\section{.}


RP11-108K3.2 $\left(P=1.986 \times 10^{-5}\right)$

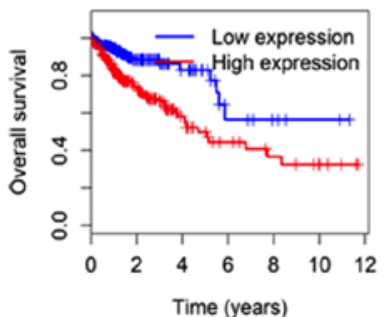

LINC01354 ( $\left(\mathrm{P}=9.856 \times 10^{-4}\right)$

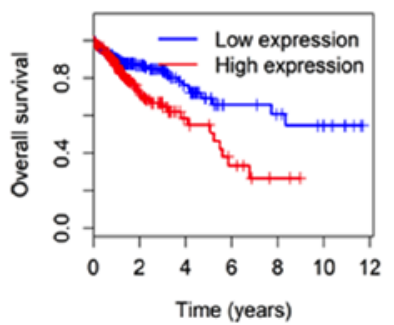

Time (years)
RP11-815M8.1 $\left(\mathrm{P}=2.785 \times 10^{-5}\right)$

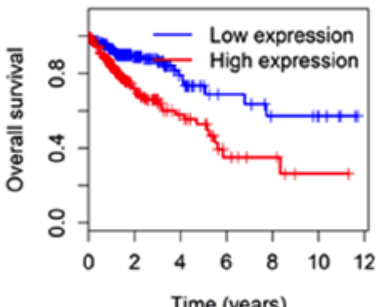

RBAKDN $(P=0.001)$

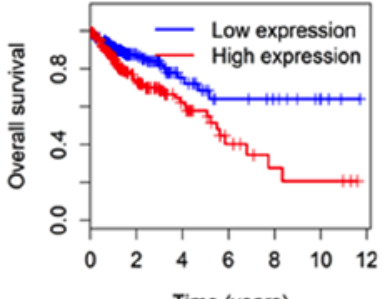

Time (years)

AFAP1-AS1 $(P=0.004)$

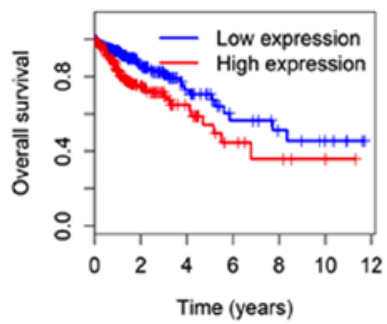

$\operatorname{LINC01836}\left(\mathrm{P}=2.569 \times 10^{-4}\right)$

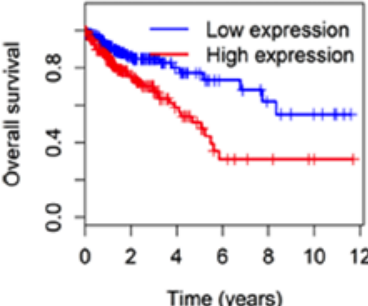

RP11-400N13.2(P=0.002)

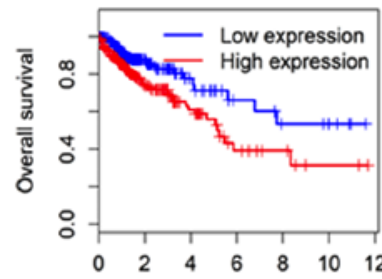

Time (years)

LINC01655 $(\mathrm{P}=0.004)$

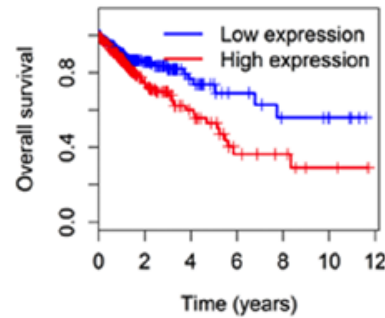

AC079612.1( $\left.P=5.357 \times 10^{-4}\right)$

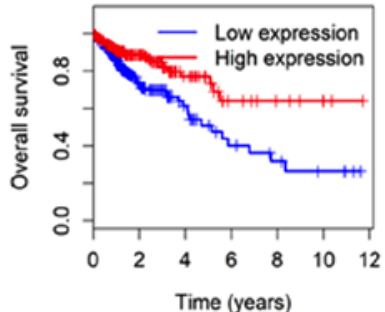

RP1-142L7.9( $P=0.004)$

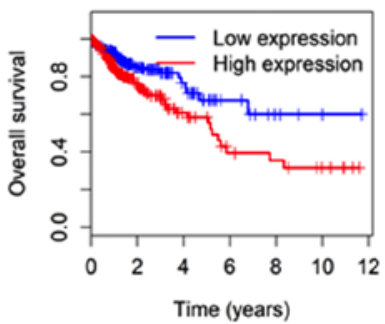

Figure 2. Top 10 lncRNAs associated with overall survival derived from 1,180 significantly differentially expressed genes. Patients were divided into high and low expression groups according to the median value of all patients. The data of the top 10 of the 56 lncRNAs are presented. lncRNA, long non-coding RNA.
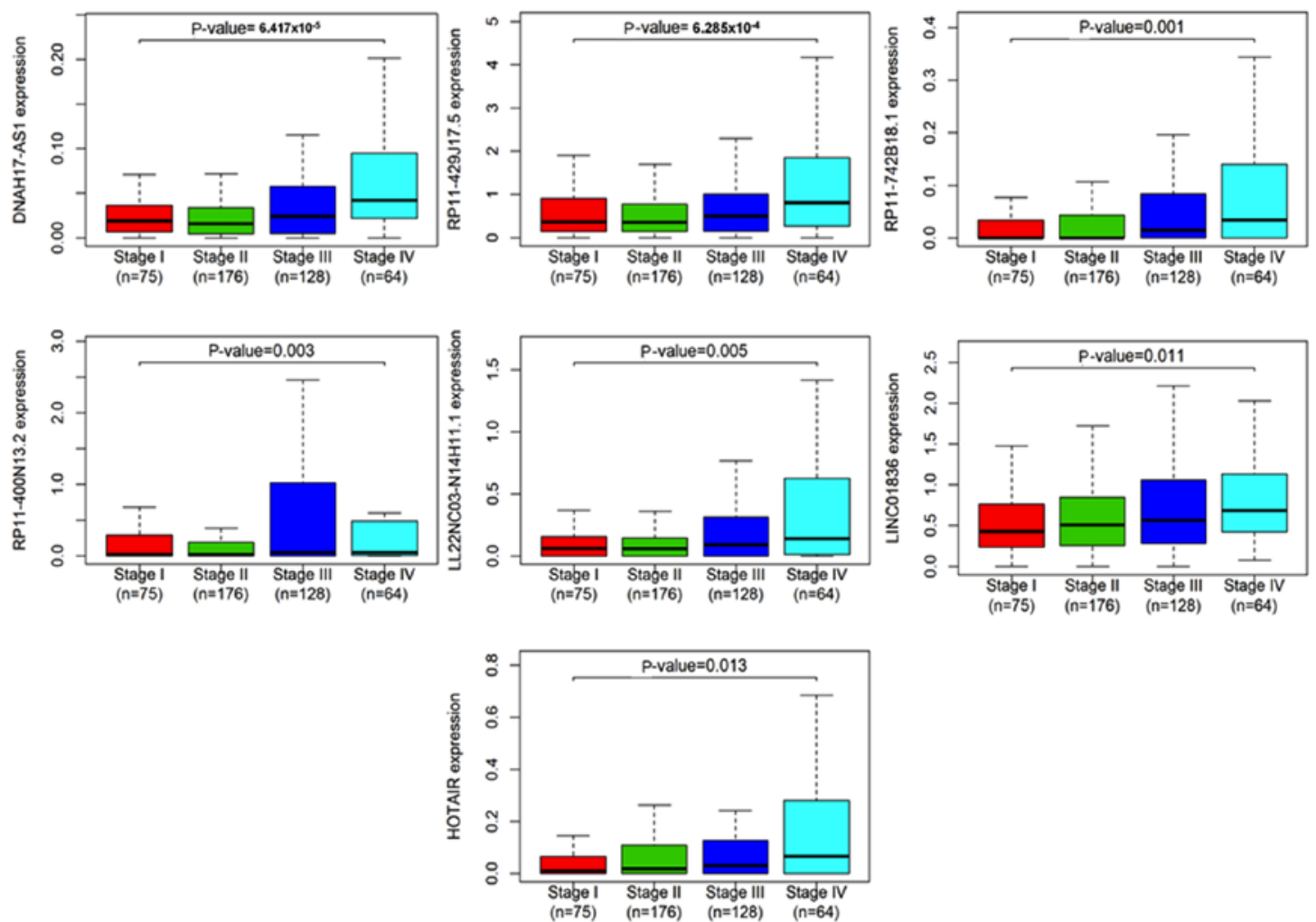

Figure 3. A total of 7 lncRNAsare significantly associated with the clinical stages of patients with colorectal cancer. The horizontal axis represents the clinical stage, whereas the vertical axis represents the expression levels of 7 lncRNAs that were significantly associated with the clinical stages of patients with colorectal cancer. IncRNA, long noncoding RNA. 

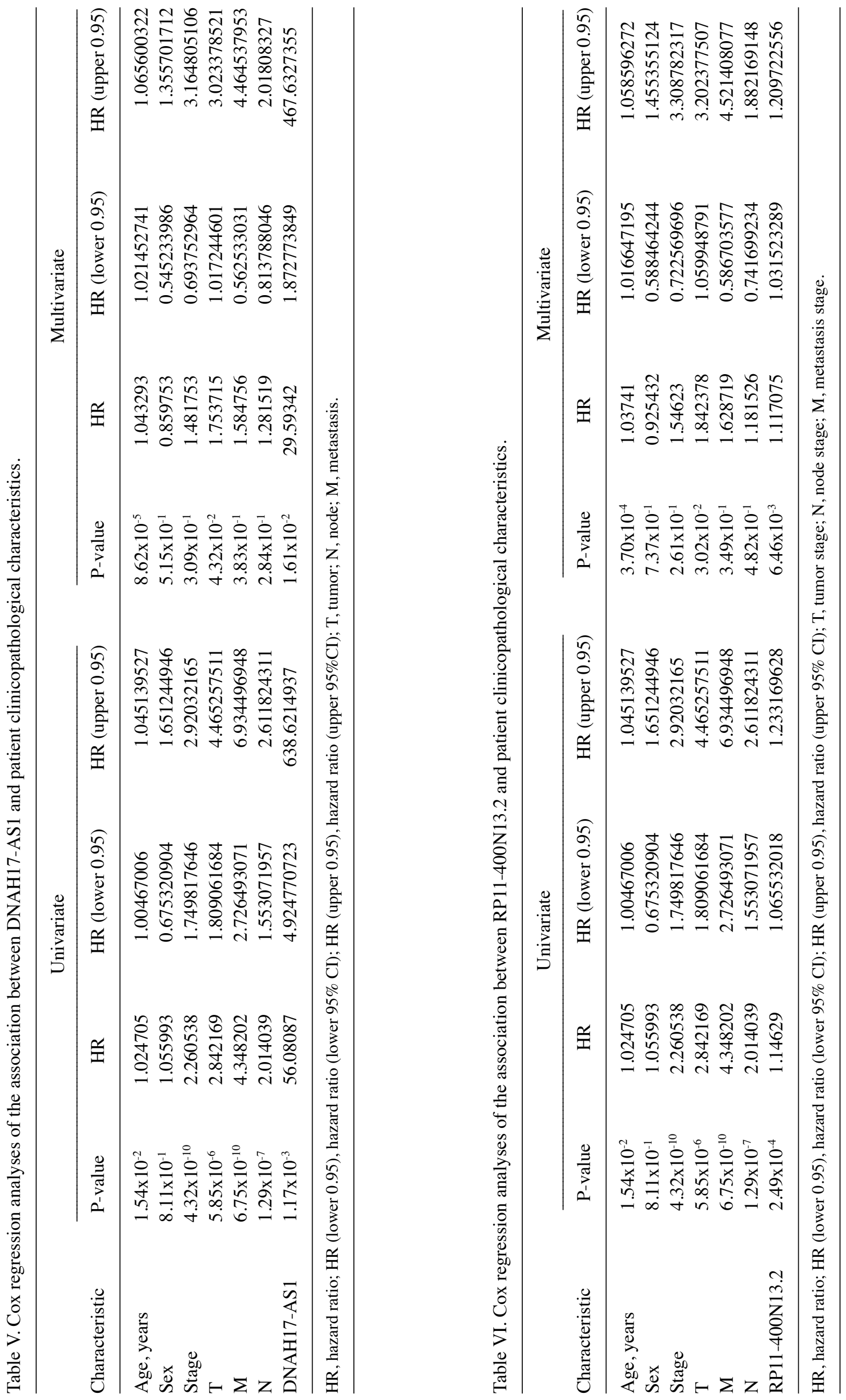
A
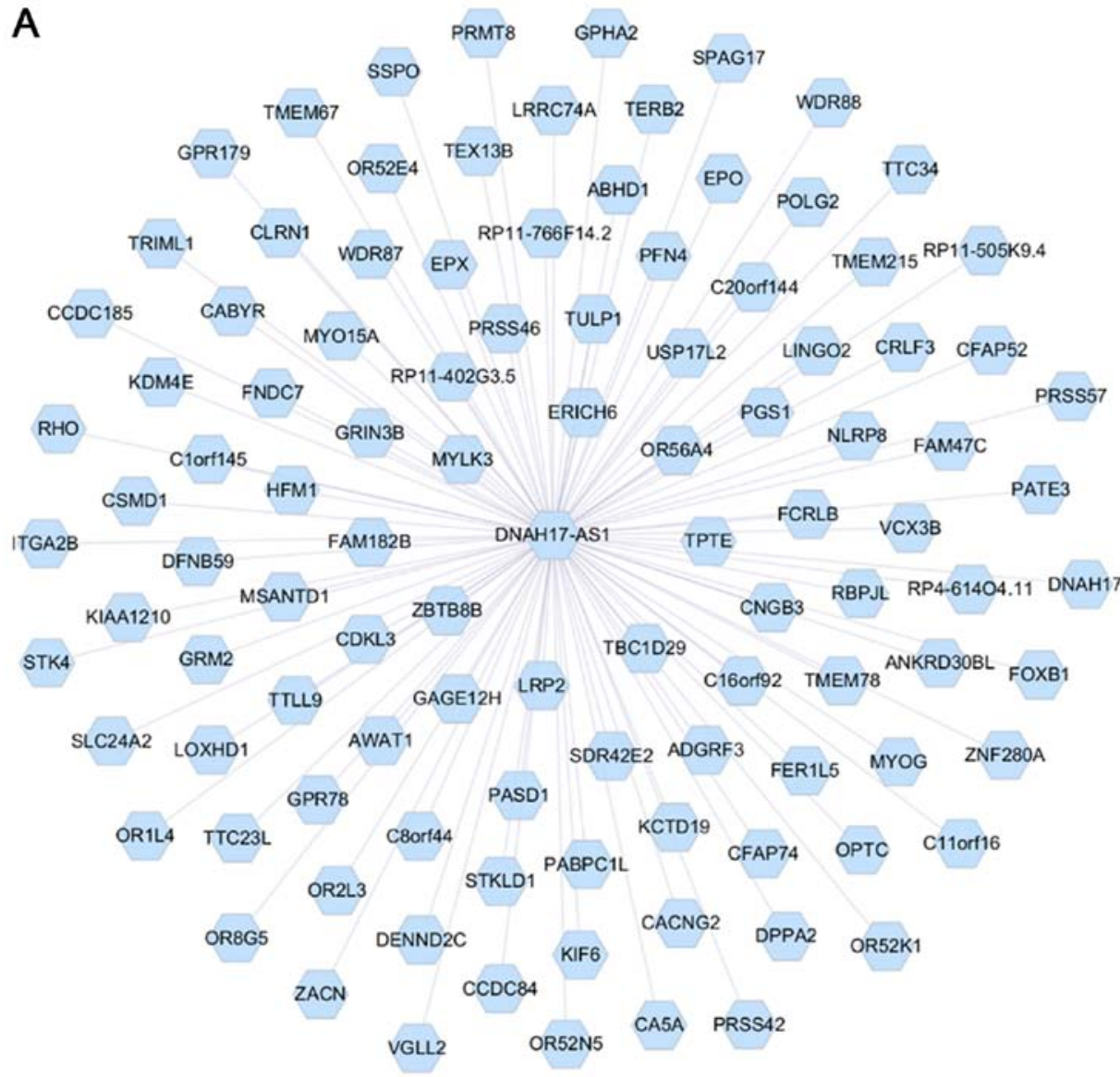

B

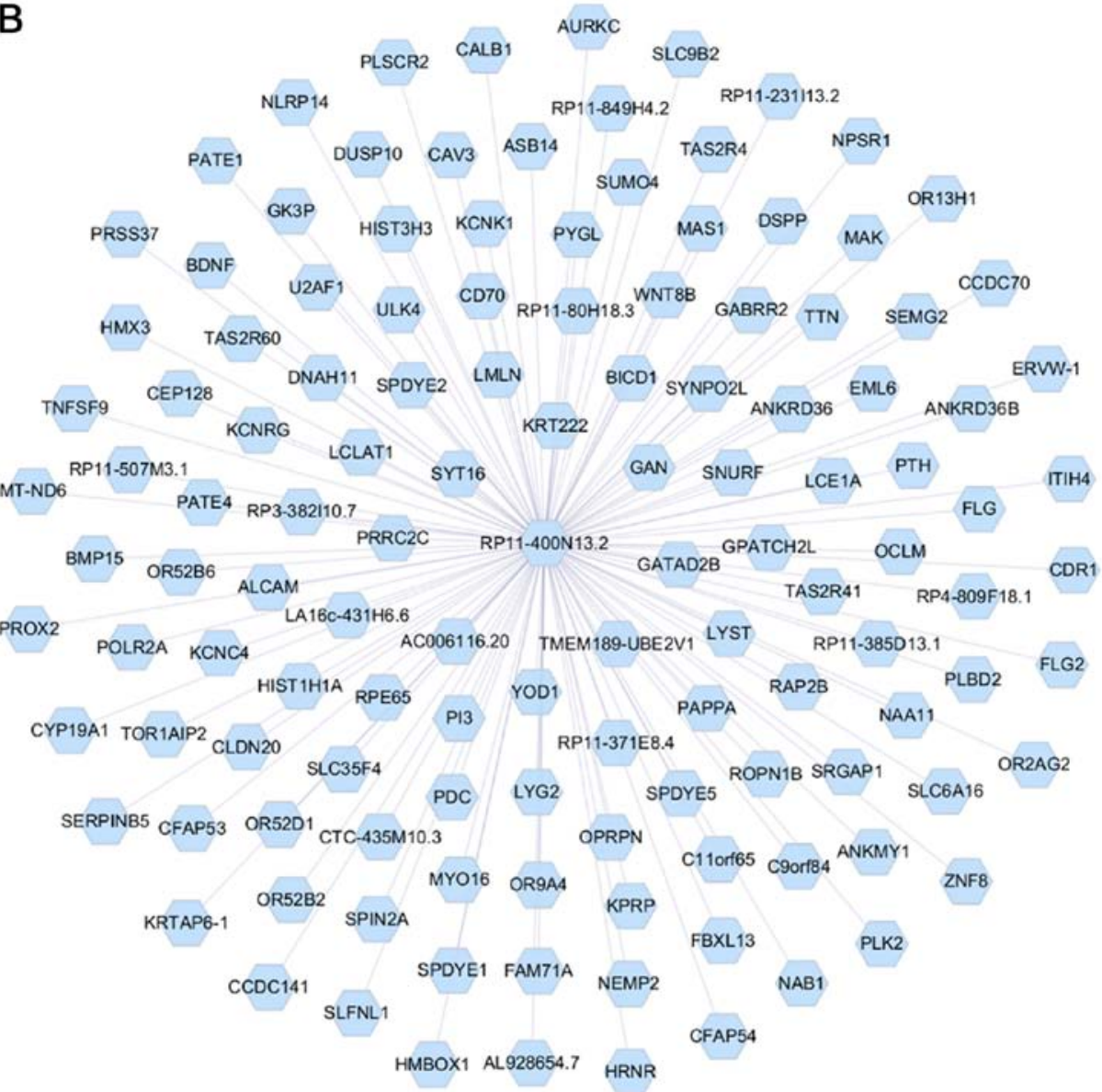

Figure 4. Interactions between two long non-coding RNAs and co-expressed PCGs. (A) DNAH17-AS1 was co-expressed with 1,048 PCGs. (B) RP11-400N13.2 was co-expressed with 126 PCGs. PCGs, protein-coding genes. 
Table VII. Co-expression analyses between DNAH17-AS1 and RP11-400N13.2 and the top 10 significant protein-coding genes.

A, Genes co-expressed with DNAH17-AS1

\begin{tabular}{lccc}
\hline $\begin{array}{l}\text { Co-expressed } \\
\text { gene }\end{array}$ & Ensembl_Gene_ID & $\mathrm{r}^{\mathrm{a}}$ & P-value \\
\hline DNAH17 & ENSG00000187775 & 0.656 & $2.73 \times 10^{-65}$ \\
SSPO & ENSG00000197558 & 0.564 & $4.89 \times 10^{-45}$ \\
TPTE & ENSG00000274391 & 0.505 & $4.31 \times 10^{-35}$ \\
DPPA2 & ENSG00000163530 & 0.473 & $1.96 \times 10^{-30}$ \\
GPR179 & ENSG00000277399 & 0.473 & $2.22 \times 10^{-30}$ \\
RP11-505K9.4 & ENSG00000260300 & 0.454 & $8.07 \times 10^{-28}$ \\
CFAP74 & ENSG00000142609 & 0.453 & $1.15 \times 10^{-27}$ \\
POLG2 & ENSG00000256525 & 0.451 & $1.99 \times 10^{-27}$ \\
KIF6 & ENSG00000164627 & 0.449 & $3.41 \times 10^{-27}$ \\
STKLD1 & ENSG00000198870 & 0.445 & $9.44 \times 10^{-27}$ \\
\hline
\end{tabular}

B, Genes co-expressed with RP11-400N13.2

\begin{tabular}{lccc}
$\begin{array}{l}\text { Co-expressed } \\
\text { gene }\end{array}$ & Ensembl_Gene_ID & $\mathrm{r}^{\mathrm{a}}$ & P-value \\
\hline RP11-371E8.4 & ENSG00000259066 & 0.439 & $5.20 \times 10^{-26}$ \\
RP11-507M3.1 & ENSG00000276087 & 0.438 & $8.64 \times 10^{-26}$ \\
HIST3H3 & ENSG00000168148 & 0.428 & $1.26 \times 10^{-24}$ \\
RP4-809F18.1 & ENSG00000255595 & 0.428 & $1.34 \times 10^{-24}$ \\
OPRPN & ENSG00000171199 & 0.426 & $2.51 \times 10^{-24}$ \\
RP11-385D13.1 & ENSG00000251537 & 0.423 & $4.79 \times 10^{-24}$ \\
OR52B2 & ENSG00000255307 & 0.422 & $5.87 \times 10^{-24}$ \\
OR52B6 & ENSG00000187747 & 0.422 & $7.24 \times 10^{-24}$ \\
OR2AG2 & ENSG00000188124 & 0.401 & $1.44 \times 10^{-21}$ \\
BDNF & ENSG00000176697 & 0.397 & $3.93 \times 10^{-21}$
\end{tabular}

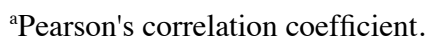

KEGG pathway enrichment analysis of the PCGs co-expressed with the two independent prognostic IncRNAs was performed using clusterProfiler package in $\mathrm{R}$ with a threshold of $\mathrm{P}<0.05$. The results revealed that the PCGs co-expressed with DNAH17-AS1 were involved in seven pathways, including 'olfactory transduction', 'neuroactive ligand-receptor interaction', 'phototransduction', 'nicotine addiction', 'cocaine addiction', 'collecting duct acid secretion' and 'signaling pathways regulating pluripotency of stem cells' (Fig. 6). This result provided novel insights into the potential associations between these pathways and CRC, which warrant further investigation. Of note, $\mathrm{P}>0.05$ was reported for the enriched pathways of the PCGs co-expressed with RP11-400N13.2.

\section{Discussion}

In the present study, in silico analysis revealed 1,180 significantly differentially expressed IncRNAs that were associated with colorectal cancer, of which 56 and 7 genes were significantly associated with OS and TNM stage, respectively. Subsequent univariate and multivariate Cox regression analyses indicated that 2 of the 7 lncRNAs, DNAH17-AS1 and RP11-400N13.2, may be independent prognostic IncRNAs for the OS of patients with colorectal cancer.

To the best of our knowledge, IncRNAs DNAH17-AS1 and RP11-400N13.2 have not been previously associated with colon cancer; however, a missense variant p.R3953Y of DNAH17, the related protein of DNAH17-AS1, was reported in undifferentiated embryonal sarcoma of the liver in a child (24). Additionally, p.R3953 of DNAH17 exhibited a high level of conservation among a variety of species, suggesting that this allele may be an important locus associated with protein function (24). A recent study revealed the mutational profile and a distinct mutation signature of $\mathrm{T}: \mathrm{A}>\mathrm{A}: \mathrm{T}$ transversion in early-stage hepatocellular carcinoma (HCC) with hepatitis B virus (HBV) infection; thus, as a key gene of the mutational profile, DNAH17 was proposed to serve an important role in the HBV-mediated transformation of liver cells (25). Additionally, the hypomethylation status of DNAH17 has been reported in HCC, which is associated with several clinical characteristics and may serve as a potential biomarker of tumor thrombosis in patients with HCC (26). In the present study, the IncRNA expression level of DNAH17-AS1 in CRC samples was analyzed and compared with that in normal samples; however, the expression level and the methylation status of DNAH17 were not analyzed. Although the expression level of DNAH17, as well as its methylation status in CRC samples, may be informative to determine the role of DNAH17 in CRC, this was beyond the scope of the present study. Therefore, relevant studies will be performed in the future.

A limited number of studies have investigated RP11-400N13.2; however, other RP11 family members have been frequently reported to be dysregulated in CRC. RP11-708H21.4, an RP11 family lncRNA located in the 17q21 gene desert region, was proposed to serve a suppressive role in the tumorigenesis of colorectal cancer and act as a novel powerful diagnostic biomarker, as well as a therapeutic target for the treatment of CRC (27). The expression levels of RP11-462C24.1, another member of the RP11 family, were determined to be significantly correlated with distant metastasis in patients with CRC, and may serve as a potential prognostic marker for such patients (28). Additionally, the dysregulation of RP11 family members has been reported to be involved in other types of cancer. For instance, a recent study revealed that overexpression of lncRNA RP11-190D6.2 inhibited the proliferation, migration and invasion of epithelial ovarian cancer (EOC) cells and may be considered a novel biomarker and therapeutic target for EOC (29). Furthermore, lncRNA RP11-436H11.5 was identified to function as a competing endogenous RNA to promote the proliferation and invasion of renal cell carcinoma (RCC) cells, which suggests that RP11-436H11.5 may be a potential therapeutic target to suppress RCC tumorigenesis (30). Collectively, the RP11 family of IncRNAs serve important roles in carcinogenesis and may be used as potential diagnostic and prognostic biomarkers for various types of cancer.

Following the identification of two independent prognostic IncRNAs in colorectal cancer, the co-expressed PCGs were 
A

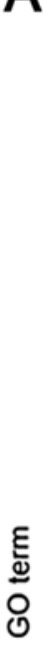

Cilium morphogenesis
G-protein coupled receptor signaling pathway, coupled to cyclic nucleotide second mensport

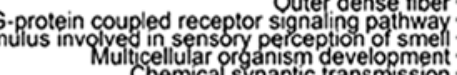

G.protein coupled photoreceptor activity.
Anion transmembrane transporter activity.

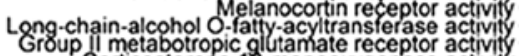

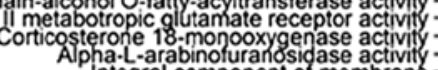

Integral integral component of membrane

Integral component of plasma membrane

Intermediate filament

Myosin filament

Photoreceptor disc membrane-

Chemical synaptic transmission Phospholipase C-activating G-protein coupled receptor sponaling pathway -

Negative regulation of cell differentiation

Anotoraceptor cell maintenance

Inorganic anion

Cell projection organization

Natural killer cell activation involved in immune response

Hair cycle

Detection of light stimulus involved Exploration behavior
Adenylate cyclase-activating serotonin receptor signaling pathway
cylation

Muscle zeil fate commitment

Glycolipid biosynthetic process

Auditory receptor cell stereocilium organization

or cell stereocilium organization

The most enriched Go terms

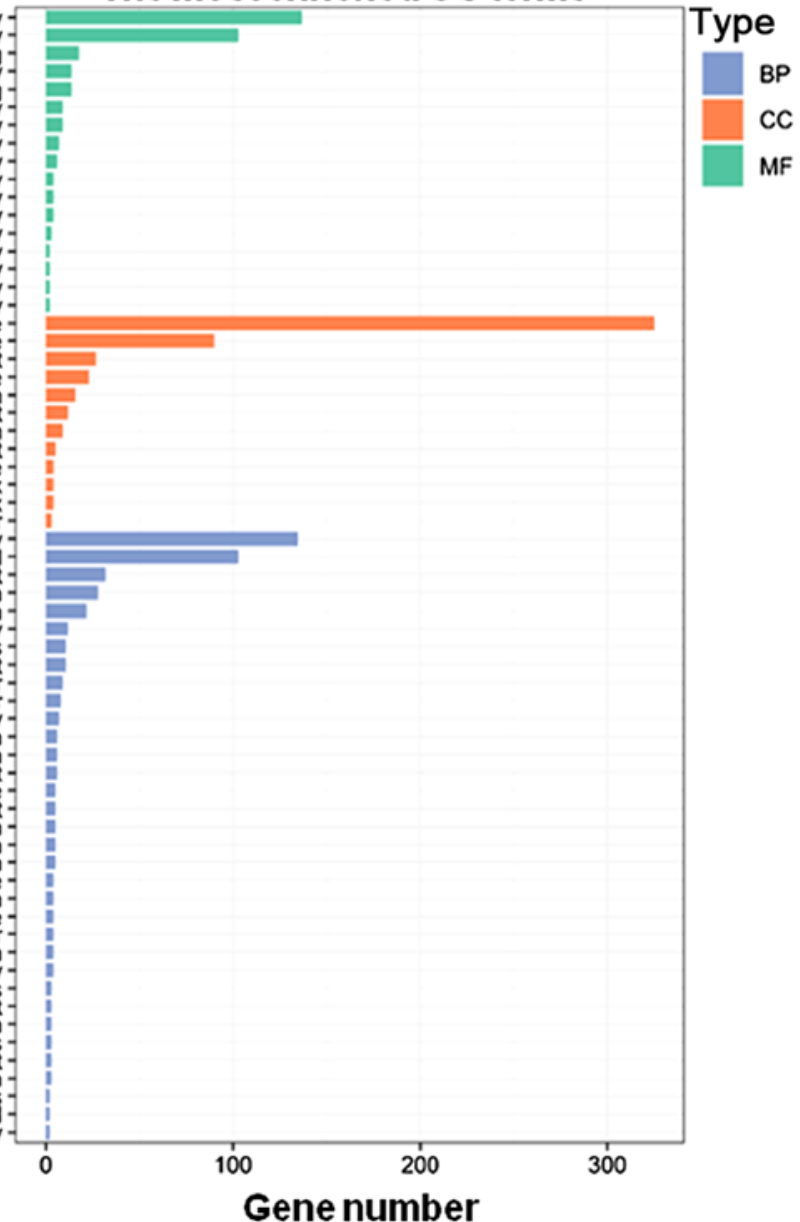

B

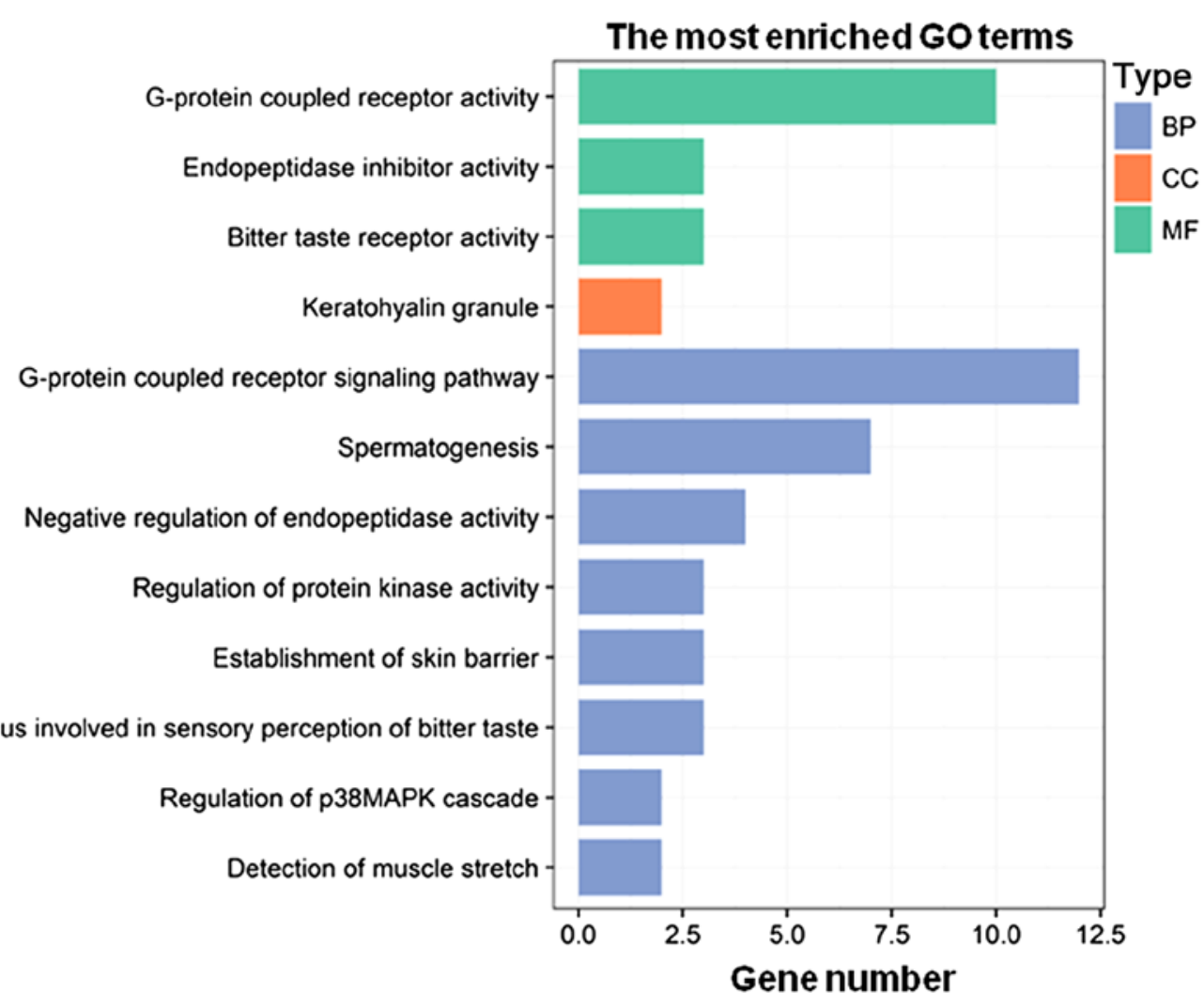

Figure 5. GO enrichment analysis maps of co-expressed PCGs associated with certain long non-coding RNAs. (A) The most enriched GO terms of PCGs co-expressed with DNAH17-AS1. (B) The most enriched GO terms of PCGs co-expressed with RP11-400N13.2. The colors of the columns represent the different types of terms. GO, Gene Ontology; PCGs, protein-coding genes; BP, biological process; CC, cellular component; MF, molecular function. 


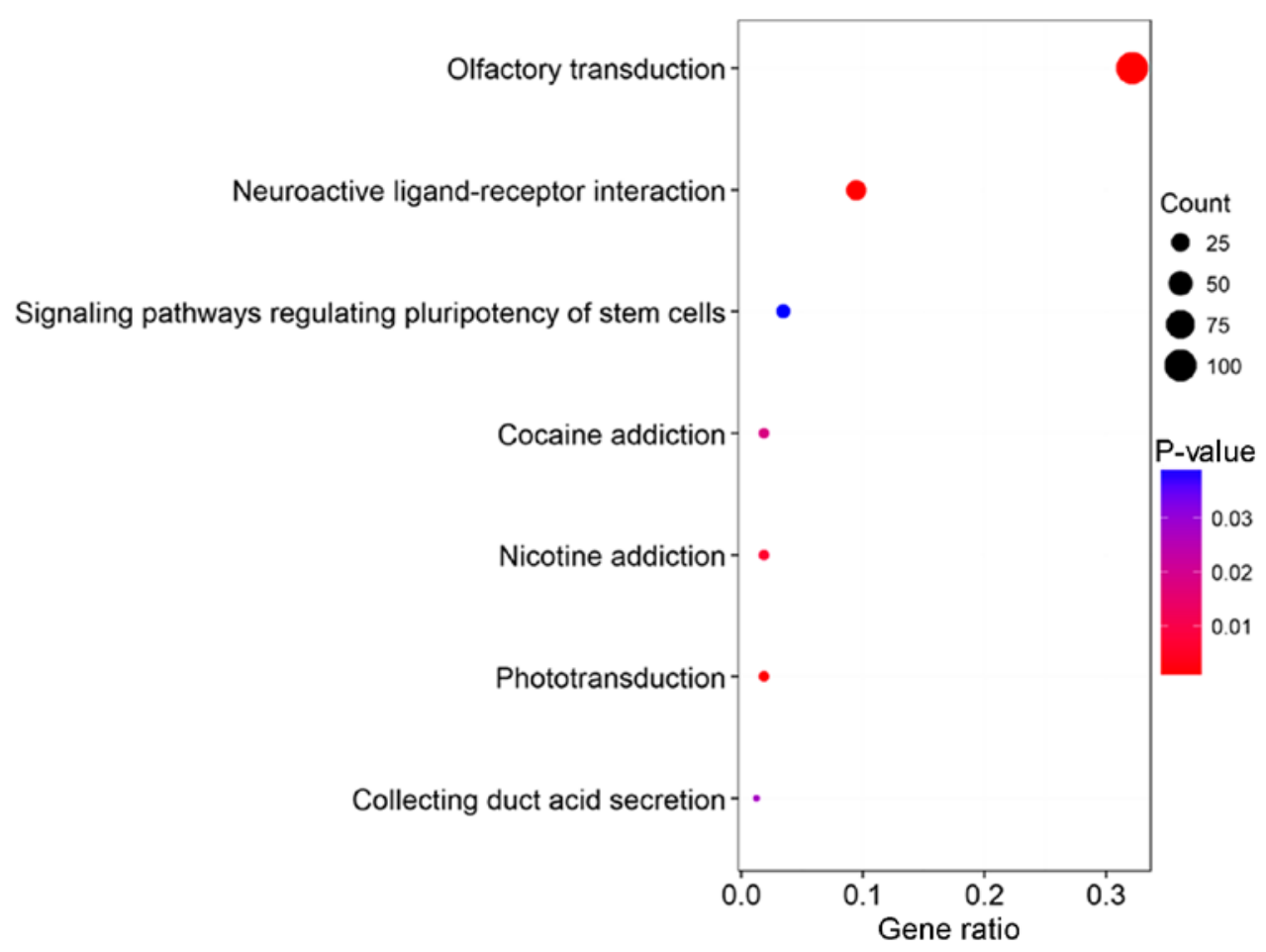

Figure 6. KEGG enrichment analysis of PCGs co-expressed with DNAH17-AS1. The size of the dots indicates the number of enriched PCGs; the color of the dots represents the degree of significance based on the P-value. KEGG, Kyoto Encyclopedia of Genes and Genomes; PCGs, protein-coding genes.

analyzed, and stepwise GO and KEGG enrichment analyses were conducted to determine the potential biological functions of these IncRNAs associated with CRC and the signaling pathways involved. The results of the functional enrichment analysis of DNAH17-AS1 and RP11-400N13.2 differed; however, these lncRNAs were determined to possess similar G-protein coupling-associated functions. G-protein coupled receptors have been previously reported to be associated with CRC tumorigenesis (31-34). For example, the G-protein coupled receptor GPR55 may promote tumor progression by acting as an pro-oncogenic factor in CRC (31). In addition, GPR55 has been proposed to be involved in the migration of CRC cells and may serve as a potential target for the prevention of metastasis (32). On the contrary, orexin receptor type 1 and cholecystokinin A receptor, which belong to family A of the G-protein coupled receptors, serve opposing roles in the regulation of HT-29 CRC cell migration, but have also been reported to be involved in the pathogenesis of CRC metastasis (33). Furthermore, a recent study revealed that GPR109A, a G-protein coupled receptor for short-chain fatty acids, was silenced in CRC cells (34). In addition, the host immune system may employ interferon $\gamma$ to counteract methylation-mediated silencing of GPR109A as a mechanism to suppress tumor development (34). Therefore, G-protein coupled receptors maybe associated with the carcinogenesis and metastasis of CRC; the roles of DNAH17-AS1 and RP11-400N13.2 in CRC, which may be mediated by these receptors, require further investigation.

In the present study, DNAH17-AS1 and RP11-400N13.2 were identified as potential independent prognostic IncRNAs for OS in patients with CRC. Further bioinformatics analyses revealed that these 2 lncRNAs may serve a pro-oncogenic role in CRC via G-protein coupling-related functions. Therefore, DNAH17-AS1 and RP11-400N13.2 may serve as prognostic biomarkers for CRC in the future. The detailed methodology of the present study is presented in Fig. S2.

\section{Acknowledgements}

Not applicable.

\section{Funding}

This study was funded by the National Natural Science Foundation of China (grant no. 81572416), the National Key Technologies R\&D Program of China (grant no. 2016YFC1303200), and the Tianjin Medical University Cancer Institute and Hospital Cancer Translational Medicine Seed Funds (grant no. 1701-1).

\section{Availability of data and materials}

The datasets used and/or analyzed during the current study are available from the corresponding author on reasonable request.

\section{Authors' contributions}

LL designed and supervised the study and finalized the manuscript. WZ and BP made substantial contributions to the study design, performed the bioinformatics analysis and drafted the manuscript. All authors read and approved the final manuscript. 


\section{Ethics approval and consent to participate}

Not applicable.

\section{Patient consent for publication}

Not applicable.

\section{Competing interests}

The authors declare that they have no competing interests.

\section{References}

1. Siegel RL, Miller KD and Jemal A: Cancer statistics, 2016. CA Cancer J Clin 66: 7-30, 2016

2. Ghareeb AE, Moawed FSM, Ghareeb DA and Kandil EI Potential prophylactic effect of berberine against rat colon carcinoma induce by 1,2-dimethyl hydrazine. Asian Pac J Cancer Prev 19: 1685-1690, 2018

3. Sanoff HK, Sargent DJ, Campbell ME, Morton RF, Fuchs CS Ramanathan RK, Williamson SK, Findlay BP, Pitot HC and Goldberg RM: Five-year data and prognostic factor analysis of oxaliplatin and irinotecan combinations for advanced colorectal cancer: N9741. J Clin Oncol 26: 5721-5727, 2008.

4. Dong Z, Zheng L, Liu W and Wang C: Association of mRNA expression of TP53 and the TP53 codon 72 Arg/Pro gene polymorphism with colorectal cancer risk in Asian population: A bioinformatics analysis and meta-analysis. Cancer Manag Res 10: 1341-1349, 2018.

5. Xue W, Li J, Wang F, Han P, Liu Y and Cui B: A long non-coding RNA expression signature to predict survival of patients with colon adenocarcinoma. Oncotarget 8: 101298-101308, 2017.

6. Dykes IM and Emanueli C: Transcriptional and posttranscriptional gene regulation by long non-coding RNA. Genomics Proteomics Bioinformatics 15: 177-186, 2017.

7. Iyer MK, Niknafs YS, Malik R, Singhal U, Sahu A, Hosono Y, Barrette TR, Prensner JR, Evans JR, Zhao S, et al: The landscape of long non-coding RNAs in the human transcriptome. Nat Genet 47: 199-208, 2015.

8. Prensner JR and Chinnaiyan AM: The emergence of lncRNAs in cancer biology. Cancer Discov 1: 391-407, 2011.

9. Zhou M, Wang X, Li J, Hao D, Wang Z, Shi H, Han L, Zhou H and Sun J: Prioritizing candidate disease-related long non-coding RNAs by walking on the heterogeneous lncRNA and disease network. Mol Biosyst 11: 760-769, 2015.

10. Lou Y, Jiang H, Cui Z, Wang X, Wang L and Han Y: Gene microarray analysis of lncRNA and mRNA expression profiles in patients with high-grade ovarian serous cancer. Int J Mol Med 42: 91-104, 2018.

11. Jin B, Jin H, Wu HB, Xu JJ and Li B: Long non-coding RNA SNHG15 promotes CDK14 expression via miR-486 to accelerate non-small cell lung cancer cells progression and metastasis. J Cell Physiol 233: 7164-7172, 2018.

12. Li S, Zhou J, Wang Z, Wang P, Gao X and Wang Y: Long noncoding RNA GAS5 suppresses triple negative breast cancer progression through inhibition of proliferation and invasion by competitively binding miR-196a-5p. Biomed Pharmacother 104 : 451-457, 2018.

13. Liu Z, Yan Y, Cao S and Chen Y: Long non-coding RNA SNHG14 contributes to gastric cancer development through targeting miR-145/SOX9 axis. J Cell Biochem 119: 6905-6913, 2018.

14. Fan H, Lv P, Mu T, Zhao X, Liu Y, Feng Y, Lv J, Liu M and Tang H: lncRNA n335586/miR-924/CKMT1A axis contributes to cell migration and invasion in hepatocellular carcinoma cells. Cancer Lett 429: 89-99, 2018.

15. Ye XT, Huang H, Huang WP and Hu WL: lncRNA THOR promotes human renal cell carcinoma cell growth. Biochem Biophys Res Commun 501: 661-667, 2018.

16. Tsai KW, Lo YH, Liu H, Yeh CY, Chen YZ, Hsu CW, Chen WS and Wang JH: Linc00659, a long noncoding RNA, acts as novel oncogene in regulating cancer cell growth in colorectal cancer. Mol Cancer 17: 72, 2018.
17. Wu Q, Meng WY, Jie Y and Zhao H: lncRNA MALAT1 induces colon cancer development by regulating miR-129-5p/HMGB1 axis. J Cell Physiol 233: 6750-6757, 2018.

18. Yang H, Wang S, Kang YJ, Wang C, Xu Y, Zhang Y and Jiang Z: Long non-coding RNA SNHG1 predicts a poor prognosis and promotes colon cancer tumorigenesis. Oncol Rep 40: 261-271, 2018.

19. Xu Y, Zhang X, Hu X, Zhou W, Zhang P, Zhang J, Yang S and Liu Y: The effects of lncRNA MALAT1 on proliferation, invasion and migration in colorectal cancer through regulating SOX9. Mol Med 24: 52, 2018.

20. Xu M, Chen X, Lin K, Zeng K, Liu X, Pan B, Xu X, Xu T, $\mathrm{Hu} \mathrm{X}$, Sun L, et al: The long noncoding RNA SNHG1 regulates colorectal cancer cell growth through interactions with EZH2 and miR-154-5p. Mol Cancer 17: 141, 2018.

21. Edge SB, Byrd DR, Compton CC, Fritz AG Greene, F and Trotti A (eds.): AJCC Cancer Staging Manual. 7th edition. Springer-Verlag, New York, NY, 2010.

22. Gene Ontology Consortium: The gene ontology (GO) project in 2006. Nucleic Acids Res 34: D322-D326, 2006.

23. Altermann E and Klaenhammer TR: Pathwayvoyager: Pathway mapping using the Kyoto encyclopedia of genes and genomes (KEGG) database. BMC Genomics 6: 60, 2005.

24. Kim JH, Sio CA, Park H, Kim H, Shin HD and Jung K: Undifferentiated embryonal sarcoma of the liver in a child: A whole exome sequencing analysis. Dig Liver Dis 49: 944-946, 2017.

25. Zhan H, Jiang J, Sun Q, Ke A, Hu J, Hu Z, Zhu K, Luo C, Ren N, Fan J, et al: Whole-exome sequencing-based mutational profiling of hepatitis B virus-related early-stage hepatocellular carcinoma. Gastroenterol Res Pract 2017: 2029315, 2017.

26. Fan X, Guo H, Dai B, He L, Zhou D and Lin H: The association between methylation patterns of DNAH17 and clinicopathological factors in hepatocellular carcinoma. Cancer Med 8: 337-350, 2019.

27. Sun L, Jiang C, Xu C, Xue H, Zhou H, Gu L, Liu Y and Xu Q: Down-regulation of long non-coding RNA RP11-708H21.4 is associated with poor prognosis of colorectal cancer and promotes tumorigenesis through regulating $\mathrm{AKT} / \mathrm{mTOR}$ pathway. Oncotarget 8: 27929-27942, 2017.

28. Shi D, Zheng H, Zhuo C, Peng J, Li D, Xu Y, Li X, Cai G and Cai S: Low expression of novel lncRNA RP11-462C24.1 suggests a biomarker of poor prognosis in colorectal cancer. Med Oncol 31: 31, 2014.

29. Tong W, Yang L, Yu Q, Yao J and He A: A new tumor suppressor lncRNA RP11-190D6.2 inhibits the proliferation, migration and invasion of epithelial ovarian cancer cells. Onco Targets Ther 10: 1227-1235, 2017.

30. Wang KF, Jin W, Song Y and Fei X: lncRNA RP11-436H11.5, functioning as a competitive endogenous RNA, upregulates BCL-W expression by sponging miR-335-5p and promotes proliferation and invasion in renal cell carcinoma. Mol Cancer 16: 166, 2017.

31. Hasenoehrl C, Feuersinger D, Sturm EM, Bärnthaler T, Heitzer E, Graf R, Grill M, Pichler M, Beck S, Butcher L, et al: G protein-coupled receptor GPR55 promotes colorectal cancer and has opposing effects to cannabinoid receptor 1. Int J Cancer 142: 121-132, 2018.

32. Kargl J, Andersen L, Hasenöhrl C, Feuersinger D, Stančić A, Fauland A, Magnes C, El-Heliebi A, Lax S, Uranitsch S, et al: GPR55 promotes migration and adhesion of colon cancer cells indicating a role in metastasis. Br J Pharmacol 173: 142-154, 2016.

33. Bai B, Chen X, Zhang R, Wang X, Jiang Y, Li D, Wang Z and Chen J: Dual-agonist occupancy of orexin receptor 1 and cholecystokinin A receptor heterodimers decreases G-protein-dependent signaling and migration in the human colon cancer cell line HT-29. Biochim Biophys Acta Mol Cell Res 1864: 1153-1164, 2017.

34. Bardhan K, Paschall AV, Yang D, Chen MR, Simon PS, Bhutia YD, Martin PM, Thangaraju M, Browning DD, Ganapathy V, et al: IFN $\gamma$ Induces DNA methylation-silenced GPR109A expression via pSTAT1/p300 and H3K18 acetylation in colon cancer. Cancer Immunol Res 3: 795-805, 2015.

This work is licensed under a Creative Commons Attribution-NonCommercial-NoDerivatives 4.0 International (CC BY-NC-ND 4.0) License. 